\title{
NOTE ON A CONVERGENCE PROOF
}

\author{
BY DUNHAM JACKSON
}

Some years ago I published a particularly simple proof of the convergence of the Fejér mean of the Fourier series for an arbitrary continuous function.* I did not notice until some time later that the same proof had already been given by Haar $\dagger$ in his thesis. The present note constitutes a renewed attempt to contribute something to the theory of the method in question, by applying it to a problem which is not treated by Haar, in the passage cited at any rate. The substance of the note consists in the proof of the following theorem: $\ddagger$

Let $f(x)$ be an arbitrary continuous function of period $2 \pi$. With each positive integral value of $n$, let an integer $m_{n}$ be associated, subject merely to the condition that $m_{n} \geqq n$, and let

$$
\tau_{n}(x)=\frac{1}{n m_{n}} \sum_{i=1}^{m_{n}} f\left(t_{i}\right) \frac{\sin ^{2} \frac{1}{2} n\left(t_{i}-x\right)}{\sin ^{2} \frac{1}{2}\left(t_{i}-x\right)},
$$

where $t_{i}=2 i \pi / m_{n}$. Then $\tau_{n}(x)$ converges uniformly toward $f(x)$ as $n$ becomes infinite.

The reasoning is given in full, so that it can be understood

* Note on a method of proof in the theory of Fourier's series, this Bulletin, vol. 27 (1920-21), pp. 108-110.

$\dagger$ A. Haar, Zur Theorie der orthogonalen Funktionensysteme, Dissertation, Göttingen, 1909 ; p. 29; reprinted in Mathematische Annalen, vol. 69 (1910), pp. 331-371; pp. 353-354.

$\ddagger$ For the case $m_{n}=n$, see D. Jackson, $A$ formula of trigonometric interpolation, Rendiconti del Circolo Matematico di Palermo, vol. 37 (1914), pp. 371-375; S. Bernstein, Sur la convergence absolue des séries trigonométriques, Comptes Rendus, vol. 158 (1914), pp. 1661-1663; L. Fejér, Über Interpolation, Göttinger Nachrichten (1916), pp. 66-91; pp. 87-91. For a corresponding generalization of the ordinary formula of trigonometric interpolation, see D. Jackson, Some notes on trigonometric interpolation, American Mathematical Monthly, vol. 34 (1927), pp. 401-405. For the underlying idea of the present treatment, see also Hahn, Über das Interpolationsproblem, Mathematische Zeitschrift, vol. 1 (1918), pp. 115-142. 
without reference to Haar's paper. By a well known identity,

$$
\begin{aligned}
& \frac{\sin ^{2} \frac{1}{2} n\left(t_{i}-x\right)}{\sin ^{2} \frac{1}{2}\left(t_{i}-x\right)}=n+2(n-1) \cos \left(t_{i}-x\right) \\
& +2(n-2) \cos 2\left(t_{i}-x\right)+\cdots+2 \cos (n-1)\left(t_{i}-x\right) \\
& \quad=n+2 \sum_{k=1}^{n-1}(n-k)\left(\cos k t_{i} \cos k x+\sin k t_{i} \sin k x\right),
\end{aligned}
$$

so that $\tau_{n}(x)$ is a trigonometric sum of order $n-1$ in $x$. Since $n-1<m_{n}$, and since

$$
\sum_{i=1}^{m_{n}} \cos k t_{i}=\sum_{i=1}^{m_{n}} \sin k t_{i}=0
$$

for $0<k<m_{n}$, it is seen that

$$
\frac{1}{n m_{n}} \sum_{i=1}^{m_{n}} \frac{\sin ^{2} \frac{1}{2} n\left(t_{i}-x\right)}{\sin ^{2} \frac{1}{2}\left(t_{i}-x\right)}=1
$$

This may also be expressed by saying that if $f(x) \equiv 1$, the corresponding $\tau_{n}(x)$ is identically equal to 1 for all values of $n$.

To take another very special case, let $f(x)$ be of the form $\cos p x$, where $p$ is a given positive integer, and let the form of the corresponding $\tau_{n}(x)$ be determined with the aid of (2). The expression $\sum_{i} \cos p t_{i} \sin k t_{i}$ is equal to zero for all values of $k$. The question ultimately at issue being one of convergence for $n=\infty$, it is sufficient to consider values of $n>2 p$. Then $p<m_{n} / 2$, and $\sum_{i} \cos ^{2} p t_{i}=m_{n} / 2$. Under the hypotheses, $p+n-1$ may or may not be less than $m_{n}$. If $p+n-1<m_{n}, \sum_{i} \cos p t_{i} \cos k t_{i}=0$ for all the values of $k$ (including $k=0$ ) that come into consideration, except $k=p$, and $\tau_{n}(x)$ reduces to a single term:

$$
\tau_{n}(x)=\frac{n-p}{n} \cos p x .
$$

If $p+n-1 \geqq m_{n}$, there is one other term, resulting from the fact that $\sum_{i} \cos p t_{i} \cos \left(m_{n}-p\right) t_{i}=m_{n} / 2$, and

$$
\tau_{n}(x)=\frac{n-p}{n} \cos p x+\frac{n-m_{n}+p}{n} \cos \left(m_{n}-p\right) x
$$


But $n-m_{n} \leqq 0$, and $\left(n-m_{n}+p\right) / n \leqq p / n$, which approaches zero as $n$ becomes infinite. So, whichever of the expressions (4), (5) may be in force from time to time as $n$ takes on successive values, it is clear that

$$
\lim _{n=\infty} \tau_{n}(x)=\cos p x,
$$

uniformly for all values of $x$. There is a corresponding proof if $f(x) \equiv \sin p x$.

On the other hand, the $\tau_{n}(x)$ corresponding to the sum of any finite number of functions is the sum of the $\tau$ 's constructed for the various functions separately, and converges if each of the latter $\tau$ 's is convergent. So $\tau_{n}(x)$ converges uniformly toward $f(x)$, whenever $f(x)$ itself is identically a trigonometric sum.

In transition, it is to be noted from (1) and (3) that $\left|\tau_{n}(x)\right| \leqq M$, if $M$ is the maximum of $|f(x)|$.

Finally, let $f(x)$ be an arbitrary continuous function of period $2 \pi$. Let $\epsilon$ be an arbitrary positive quantity. By Weierstrass's theorem there exists a trigonometric sum $T(x)$ such that

$$
|f(x)-T(x)| \leqq \epsilon / 3
$$

for all values of $x$. If $\tau_{n}(x)$ is defined by (1), and if $\tau_{n 1}(x)$ is similarly formed with $T\left(t_{i}\right)$ in place of $f\left(t_{i}\right)$, it follows from the preceding paragraph, applied to the difference $T(x)-f(x)$, that

$$
\left|\tau_{n 1}(x)-\tau_{n}(x)\right| \leqq \epsilon / 3
$$

for all values of $n$ and $x$. And by the italics at the end of the second paragraph preceding,

$$
\left|T(x)-\tau_{n 1}(x)\right| \leqq \epsilon / 3
$$

if $n$ is sufficiently large. For such values of $n$, by combination of (6), (7), and (8), $\left|f(x)-\tau_{n}(x)\right| \leqq \epsilon$, which is equivalent to the conclusion of the theorem.

The University of Minnesota 